\title{
MIGRACIÓN PARA ESTUDIAR EN ESCUELAS DE MEDICINA HUMANA EN PERÚ
}

\author{
Diego Chambergo-Michilot (10,a, Carlos E. Muñoz-Medina(10) 2,b, \\ Diego Lizarzaburu-Castagnino (1D) ${ }^{1, b}$, Franco León-Jiménez (iD) ${ }^{3,4, c}$, Miguel Odar-Sampé (iD) 5,b, \\ Reneé Pereyra-Elías (iD ${ }^{6, c}$, Percy Mayta-Tristán (iD) 1,5,7,d, Red LIRHUS* \\ ${ }^{1}$ Escuela de Medicina, Universidad Científica del Sur, Lima, Perú. \\ ${ }^{2}$ Universidad de Oriente - Núcleo de Bolívar, Bolívar, Venezuela. \\ ${ }^{3}$ Universidad Católica Santo Toribio de Mogrovejo, Chiclayo, Perú. \\ ${ }^{4}$ Hospital Regional de Lambayeque, Chiclayo, Perú. \\ ${ }^{5}$ Universidad Privada Antenor Orrego, Trujillo, Perú. \\ ${ }^{6}$ Escuela de Medicina, Universidad Peruana de Ciencias Aplicadas, Lima, Perú. \\ ${ }^{7}$ Dirección General de Investigación, Desarrollo e Innovación, Universidad Científica del Sur, Lima, Perú. \\ ${ }^{\mathrm{a}}$ Estudiante de medicina; ${ }^{\mathrm{b}}$ médico cirujano; ${ }^{\mathrm{c}}$ médico epidemiólogo; ${ }^{\mathrm{d}}$ médico salubrista. \\ * Grupo Colaborativo Latinoamericano para la Investigación en Recursos Humanos en Salud (Red-LIRHUS).
}

\section{RESUMEN}

Con el objetivo de caracterizar el perfil del estudiante de medicina migrante de primer y quinto año de 32 escuelas de medicina del Perú, se realizó un análisis secundario del estudio Red-LIRHUS (2011-2012). De los 3680 estudiantes peruanos incluidos, uno de cada cuatro $(23,2 \%)$ fueron migrantes. Menos de $1 \%$ fueron migrantes internacionales. Hubo mayor proporción de estudiantes migrantes en universidades de provincias que en universidades de Lima (27,1\% vs. 15,8\%). Asimismo, hubo mayor proporción de migrantes en universidades privadas $(28,3 \%$ vs. $16,0 \%)$. Los estudiantes migrantes tenían mayor probabilidad de vivir solos (37,4\% vs. 6,4\%) y de haber desaprobado cursos (51,0\% vs. 38,6\%) en comparación con los estudiantes no migrantes. Es necesario evaluar potenciales intervenciones para la preservación del bienestar de las personas que migran para su formación médica.

Palabras clave: Emigración e Inmigración; Migración Interna; Estudiantes de Medicina; Perú (fuente: DeCS BIREME)

\section{MIGRATION TO STUDY IN MEDICAL SCHOOLS OF PERU}

\begin{abstract}
The aim of this study was to describe the frequency of migration to study medicine in Peru. We conducted a secondary data analysis of the Red-LIRHUS study (2011-2012). We included 3680 Peruvian students. Approximately, $23.2 \%$ migrated for medical school. Less than $1 \%$ were international migrants. We found a higher proportion of migrant students in Universities outside of Lima than in Universities in Lima (27.1\% vs. $15.8 \%)$. There was also a higher proportion of migrants in private universities (28.3\% vs. $16.0 \%)$ Migrant students were more likely to live alone (27.4\% vs. $6.4 \%)$ and to report having failed a module/course (51.0\% vs. $38.6 \%$ ) compared to non-migrant students. It is necessary to evaluate potential interventions for the preservation of the well-being of people who migrate for their medical training.
\end{abstract}

Citar como: Chambergo-Michilot D, Muñoz-Medina CE, Lizarzaburu-Castagnino D, León-Jiménez F, Odar-Sampé M, Pereyra-Elías R, et al. Migración para estudiar en escuelas de Medicina Humana del Perú. Rev Peru Med Exp Salud Publica. 2020;37(1):81-6. Doi: https://doi.org/10.17843/rpmesp.2020.371.4695

Correspondencia: Percy MaytaTristán; Av. Panamericana Sur Km19, Lima,Perú; pmayta@cientifica.edu.pe

Recibido: $27 / 03 / 2019$

Aprobado: 30/12/2019

En línea: 23/03/2020
Keywords: Emigration and Immigration; Human Migration; Medical Students; Peru (source: MeSH NLM)

\section{INTRODUCCIÓN}

La migración es un fenómeno demográfico mundial basado en el desplazamiento a otras ciudades o países para residir, usualmente persiguiendo mejores condiciones de vida, trabajo o estudio. Se estima que el 3,3\% de la población mundial es migrante internacional ${ }^{(1)}$, y América Latina se encuentra en un escenario de alta movilidad debido a la crisis política y social en Venezuela, donde países que tradicionalmente emitían migrantes internacionales ahora son receptores como el caso de Perú ${ }^{(2)}$. Sin embargo, el Perú tiene una alta tradición de migración interna, se estima que al menos $20 \%$ de peruanos viven en un departamento distinto al que han nacido, con un fuerte movimiento de zonas rurales a las ciudades, y de zonas de selva y sierra hacia la costa ${ }^{(3)}$. 
Uno de los factores condicionantes para la migración es la búsqueda de un desarrollo académico y profesional, por lo que también se migra para estudiar una carrera, siendo los principales destinos las ciudades con universidades. Esta movilidad interna depende de un nivel mínimo de acceso económico para costear por lo menos residencia y alimentación fuera de la ciudad de origen, si estudia en una universidad pública, o mayor aún si lo hace en una universidad privada. El Estado peruano creó en 2011 un programa denominado Beca 18, que brinda la oportunidad a estudiantes de secundaria en situación de pobreza o pobreza extrema a estudiar en institutos tecnológicos y universidades públicas y privadas cubriendo la totalidad de los $\operatorname{costos}^{(4)}$; sin embargo, no incluía la carrera de medicina, hasta la reciente convocatoria 2019.

Dado que se requieren evidencias que ayuden a implementar estrategias para un mejor resultado de los futuros estudiantes de medicina, es importante conocer cuál es el perfil de los estudiantes de medicina en función a si han migrado. Por ello, este estudio tiene como objetivo caracterizar el perfil del estudiante de medicina migrante de primer y quinto año de 32 escuelas de medicina del Perú.

\section{EL ESTUDIO}

Se realizó un estudio transversal a partir de los datos del estudio Red-LIRHUS. El estudio original evaluó entre septiembre de 2011 y julio de 2012, a 11072 estudiantes de primer y quinto año de medicina humana de 63 universidades en once países latinoamericanos hispanohablantes (Perú, Bolivia, Chile, Colombia, Costa Rica, Ecuador, El Salvador, Honduras, México, Paraguay y Venezuela). La metodología de este estudio ha sido reportada a detalle previamente ${ }^{(5)}$.

Se evaluó la frecuencia de estudiantes que reportó haber migrado para estudiar la carrera (se comparó la frecuencia de Perú con los otros diez países incluidos). Asimismo, realizamos análisis adicionales en los estudiantes de universidades de Perú donde se incluyeron el total de las 32 escuelas de medicina humana existentes al momento de recolección de datos (censo). El estudio original fue aprobado por el Comité de Ética en Investigación del Instituto Nacional de Salud del Perú (223-2011-CIEI/INS), y todos los participantes firmaron un consentimiento informado previo al llenado de las encuestas.

Para definir la variable migración, consideramos a los participantes como «migrantes» (sí o no) si el lugar donde culminó los estudios secundarios fue diferente al lugar donde cursaba los estudios de pregrado de medicina. Esta migración fue clasificada, a su vez, como nacional o internacional. Asimismo, evaluamos si fueron migrantes «de provincia a provincia», «de provincia a capital» $\mathrm{o}$ «de capital a provincia».

\section{MENSAJES CLAVE}

Motivación para realizar el estudio: Beca 18 está financiando la carrera de medicina y existe escasa evidencia sobre el perfil migratorio de estudiantes de medicina en el Perú.

Principales hallazgos: Uno de cada cuatro estudiantes de medicina peruanos es migrante y estudia, con mayor frecuencia, en universidad de provincias y en universidades privadas. En comparación con estudiantes no migrantes, los migrantes con mayor frecuencia viven solos y tienen resultados académicos desfavorables.

Implicancias: Se debe evaluar la implementación de programas de acompañamiento (académico/salud mental) a estudiantes migrantes.

Las variables empleadas para caracterizar a los participantes fueron las siguientes: género (varón o mujer), personas con quien vive (padres, familiares, amigos o solo), padres migrantes (ambos, uno o ninguno), familiares médicos (sí o no), año de estudios (primero o quinto), lugar de universidad (capital o provincia) y financiamiento de la universidad (pública o privada), así como nivel educativo de los padres (universitario o posgrado) como un proxy de acceso económico (ambos, uno o ninguno). Además, se incluyó variables relacionadas al avance y satisfacción con la carrera: desaprobación de al menos un curso (sí o no), satisfacción con la carrera (sí o no; de la respuesta a ¿usted está satisfecho con estudiar la carrera de medicina?: satisfecho y muy satisfecho vs indiferente, insatisfecho y muy insatisfecho), intención de retirarse de la carrera (sí o no; ¿̇ha pensado en algún momento retirarse o cambiar de carrera?).

Para el análisis estadístico, se utilizó el paquete estadístico Stata 13.0 (Stata Corporation, College Station, Texas, USA). Se reportaron las frecuencias y porcentajes de las variables incluidas. En el análisis de los estudiantes en el Perú, se compararon las características de quienes reportaron haber migrado para estudiar la carrera con quienes no lo hicieron, mediante la prueba Chi cuadrado, previa comprobación de los valores esperados. En vista de que el tamaño de muestra se encuentra en el orden de millares de participantes, consideramos un valor de $\mathrm{p}<0,01$ como estadísticamente significativo.

\section{HALLAZGOS}

\section{Frecuencia de estudiantes migrantes en el estudio RED-LIRHUS}

De los 11072 estudiantes (52,7\% mujeres, 20,4 años), 3680 fueron estudiantes de universidades peruanas (50,9\% mujeres, 20,5 años). En toda la muestra, el 32,8\% reportó haber 
migrado para estudiar la carrera. Esta proporción varió de forma importante entre países. Perú fue el país donde menos estudiantes reportaron haber migrado para estudiar (23,2\%). Mientras tanto, en Bolivia, Costa Rica y El Salvador, la frecuencia de estudiantes migrantes se encontraba alrededor del 50\% (Figura 1).

\section{Caracterización de los estudiantes migrantes en el Perú}

En las universidades de Lima, el 15,8\% de los estudiantes fueron migrantes ( $15,3 \%$ nacionales y $0,5 \%$ internacionales). Mientras tanto, en las universidades de provincia, el 27,1\% fueron migrantes (20,5\% de otras provincias, $6,0 \%$ de Lima y $0,6 \%$ internacionales). En Lima y provincias, la proporción de estudiantes que reportaron haber migrado del extranjero fue baja $(<1 \%)$.

Las características de los estudiantes migrantes y no migrantes fueron similares en función al género, año de estudio o tener familiares médicos. Los estudiantes migrantes reportaron, con mayor frecuencia, que uno o ambos padres fueron también migrantes $(\mathrm{p}<0,001)$; así mismo tenían, en mayor proporción, padres (uno o ambos) con estudios universitarios y de posgrado $(\mathrm{p}=0,006)$. Los estudiantes migrantes reportaron más frecuentemente vivir solos en comparación con los no migrantes ( $37,4 \%$ vs. $6,4 \% ; \mathrm{p}<0,001)$.

En relación con la universidad de destino, fue mayor la proporción de migrantes en universidades de provincia y en universidades privadas (Tabla 1). Respecto a resultados académicos, el 51,0\% de estudiantes migrantes desaprobó al menos un curso, mientras que esta proporción fue 38,6\% en estudiantes no-migrantes $(\mathrm{p}<0,001)$. La insatisfacción con la carrera fue ligeramente más alta en el grupo migrante ( $13,9 \%$ vs. $10,0 \% ; \mathrm{p}=0,001)$, así como la consideración de retirarse de la carrera $(26,0 \%$ vs. $21,0 \%$; $\mathrm{p}=0,002)$.

\section{DISCUSIÓN}

Según los resultados del estudio, uno de cuatro estudiantes de escuelas de medicina humana de Perú reportó haber migrado para estudiar la carrera, mientras que en otros países latinoamericanos incluidos en el estudio Red-LIRHUS fue hasta $50 \%$. La proporción de migrantes en universidades localizadas en Lima fue $15,8 \%$, mientras que en provincias fue casi el doble. La proporción de estudiantes migrantes en Lima fue algo más baja que lo reportado en otros estudios realizados en algunas escuelas de medicina del país. Pereyra-Elías et al. observaron que la proporción de estudiantes migrantes nacionales de carreras de Ciencias de la Salud de una universidad privada en Lima fue $19,6 \%{ }^{(6)}$. Otro estudio reportó que la cifra de estudiantes migrantes nacionales de segundo año de una universidad de Lima fue $36,7 \%$, y que $17,2 \%$ provino de ciudades pequeñas ${ }^{(7)}$. Por otro lado, la proporción de estudiantes extranjeros en nuestro estudio fue baja. Estudios en Alemania reportan alrededor de 10\% de estudiantes extranjeros en escuelas de medicina ${ }^{(8,9)}$. En Chile, se registró que la cifra de universitarios extranjeros fue $0,4 \%{ }^{(10)}$, similar a la reportada en el presente estudio. La diferencia de la migración internacional entre Alemania

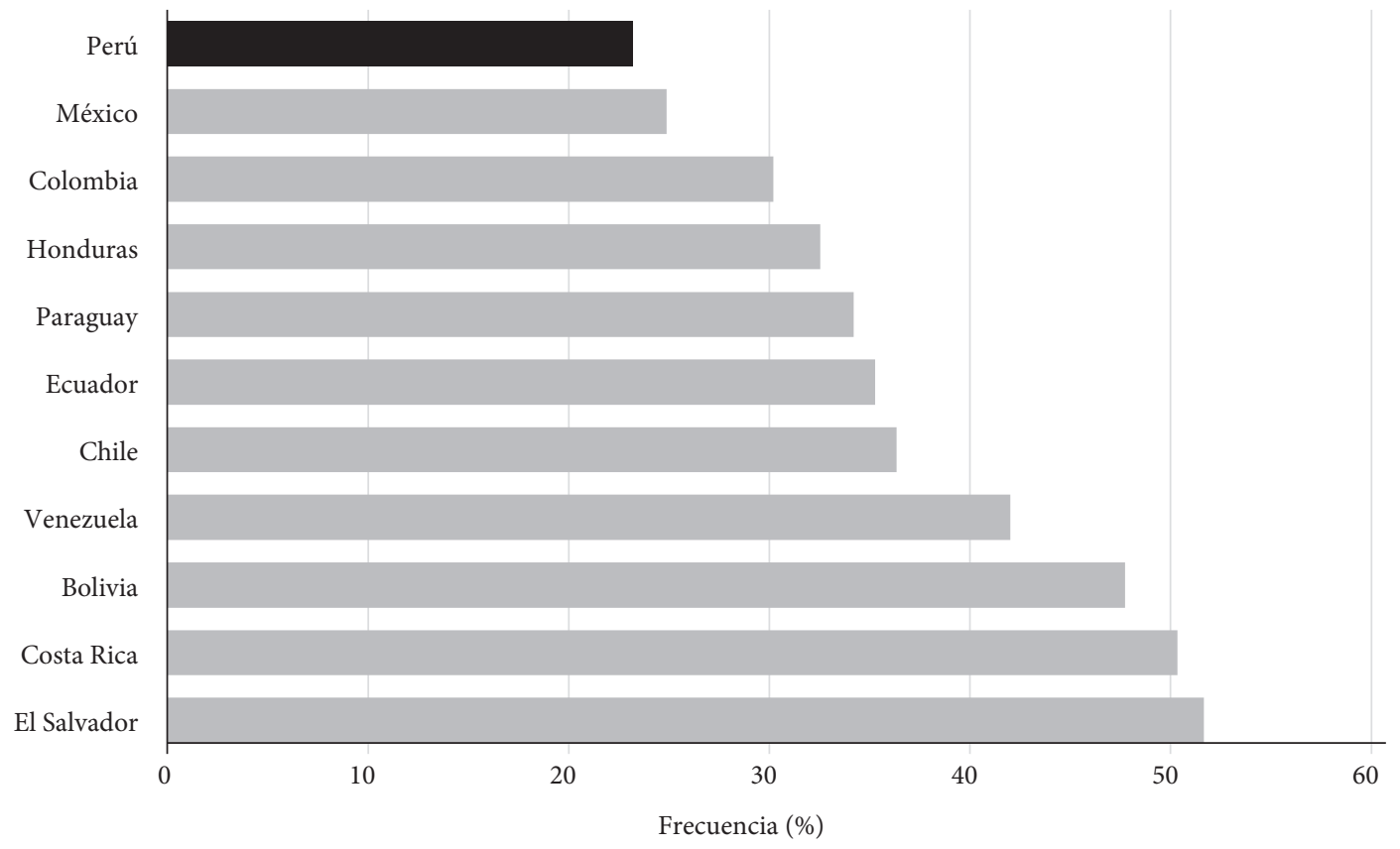

Figura 1. Frecuencia de migración para estudiar la carrera en estudiantes de medicina de 11 países de Latinoamérica, estudio Red-LIRHUS, 2011-2012. 
Tabla 1. Características sociodemográficas y académicas de estudiantes de medicina peruanos según condición de migración, LIRHUS 2011$2012\left(n=3680^{a}\right)$.

\begin{tabular}{|c|c|c|c|c|c|}
\hline \multirow{3}{*}{ Características } & \multicolumn{4}{|c|}{ Migró para estudiar } & \multirow{3}{*}{ Valor de $\mathbf{p}^{b}$} \\
\hline & \multicolumn{2}{|c|}{ Sí } & \multicolumn{2}{|c|}{ No } & \\
\hline & $\mathbf{n}$ & $(\%)$ & $\mathbf{n}$ & $(\%)$ & \\
\hline \multicolumn{6}{|l|}{ Género } \\
\hline Varón & 445 & 52,2 & 1360 & 48,2 & 0,041 \\
\hline Mujer & 408 & 47,8 & 1463 & 51,8 & \\
\hline \multicolumn{6}{|l|}{ Año } \\
\hline Primero & 554 & 64,9 & 1820 & 64,4 & 0,802 \\
\hline Quinto & 300 & 35,1 & 1006 & 35,6 & \\
\hline \multicolumn{6}{|c|}{ Lugar de universidad } \\
\hline Lima & 202 & 23,7 & 1064 & 37,7 & $<0,001$ \\
\hline Provincias & 652 & 76,3 & 1762 & 62,3 & \\
\hline \multicolumn{6}{|c|}{ Tipo de universidad } \\
\hline Pública & 240 & 28,3 & 1272 & 45,1 & $<0,001$ \\
\hline Privada & 609 & 71,7 & 1547 & 54,9 & \\
\hline \multicolumn{6}{|l|}{ Vive con } \\
\hline Padres & 359 & 42,4 & 2421 & 86,8 & $<0,001$ \\
\hline Familiares & 123 & 14,5 & 115 & 4,1 & \\
\hline Amigos & 48 & 5,7 & 75 & 2,7 & \\
\hline Solo & 316 & 37,4 & 179 & 6,4 & \\
\hline \multicolumn{6}{|l|}{ Padres migrantes } \\
\hline Ambos & 568 & 67,5 & 714 & 25,9 & $<0,001$ \\
\hline Uno & 192 & 22,8 & 835 & 30,3 & \\
\hline Ninguno & 82 & 9,7 & 1208 & 43,8 & \\
\hline \multicolumn{6}{|c|}{ Padres universitarios } \\
\hline Ambos & 408 & 47,8 & 1177 & 41,6 & 0,006 \\
\hline Uno & 220 & 25,8 & 787 & 27,9 & \\
\hline Ninguno & 226 & 26,4 & 862 & 30,5 & \\
\hline \multicolumn{6}{|c|}{ Familiares médicos } \\
\hline Sí & 458 & 53,8 & 1414 & 50,3 & 0,072 \\
\hline No & 393 & 46,2 & 1397 & 49,7 & \\
\hline \multicolumn{6}{|c|}{ Desaprobó un curso } \\
\hline Sí & 429 & 51,0 & 1070 & 38,6 & $<0,001$ \\
\hline No & 412 & 49,0 & 1702 & 61,4 & \\
\hline \multicolumn{6}{|c|}{ Insatisfecho con la carrera } \\
\hline Sí & 118 & 13,9 & 279 & 10,0 & 0,001 \\
\hline No & 734 & 86,1 & 2519 & 90,0 & \\
\hline \multicolumn{6}{|c|}{ Pensó en retirarse de la carrera } \\
\hline Sí & 214 & 26,0 & 569 & 21,0 & 0,002 \\
\hline No & 608 & 74,0 & 2144 & 79,0 & \\
\hline
\end{tabular}

a Algunas variables tienen valores perdidos; ${ }^{\text {b }}$ obtenido mediante la prueba Chi cuadrado

con Chile y Perú se debe a que las universidades mejor posicionadas en rankings internacionales son más atractivas para estudiantes internacionales con mayores recursos; pero otros factores son la gratuidad de la enseñanza y la facilidad de ingreso. Las universidades públicas en Chile no son gratuitas y el ingreso en las peruanas es muy selectivo en medicina, llegando a 50 postulantes por cada vacante en el caso de la Universidad Nacional Mayor de San Marcos. Un caso diferente en Latinoamérica se ve en Argentina, donde la Facultad de Medicina de la Universidad de Buenos Aires ${ }^{(11)}$ tiene 5,7\% de estudiantes internacionales y, a diferencia de Perú y Chile, es gratuita y su ingreso no es tan complicado.
La mayor proporción de estudiantes migrantes internos es de provincia a otras provincias, lo que puede deberse a las dificultades que supone dejar a los familiares en la ciudad de origen por migrar para estudiar ${ }^{(4)}$. Esto podría llevar a los aspirantes a escoger universidades cercanas (en otras provincias) para visitar a sus familiares con mayor facilidad y frecuencia. Por otro lado, el mayor costo de vida que representa vivir en la capital peruana podría también influir en la decisión ${ }^{(12)}$. Asimismo, existe una mayor proporción de estudiantes migrantes internos en universidades privadas, que podría deberse a que la migración implica un costo que no es accesible a toda la población por igual, sino a estu- 
diantes de familias con mayores ingresos, que son hijos de padres con estudios universitarios y de posgrado. Por último, la baja proporción de migrantes internacionales podría deberse a una combinación de los factores anteriormente descritos, sumado a que pocas universidades peruanas figuran en los rankings internacionales de calidad académica/ investigación ${ }^{(13)}$.

La cantidad de estudiantes migrantes, tanto nacionales como internacionales, que viven solos es aproximadamente seis veces más alta que los no migrantes, además tuvieron mayor probabilidad de desaprobar, lo cual se asocia más a vivir solo que a la migración per se. Los migrantes presentan mayor probabilidad de enfrentar soledad y problemas de salud mental ${ }^{(8,14-17)}$, lo cual podría estar asociado a resultados académicos desfavorables ${ }^{(9)}$. Hunh D et al. observaron que los estudiantes de medicina autóctonos de Alemania presentaron resultados académicos superiores en comparación a los migrantes no europeos ${ }^{(9)}$. Por el contrario, otro estudio (6) en estudiantes peruanos de ciencias de la salud encontró que ser migrante no se asoció a síntomas depresivos, pero sí a estar inconforme con la situación económica actual.

Los migrantes afrontan diversos retos en el proceso de aculturación, principalmente la soledad social (sentimientos subjetivos de soledad) y vivir solo ${ }^{(15,16)}$, esto último debido al alejamiento familiar y de sus círculos sociales. A pesar de que en el presente estudio no se reportan otros factores asociados a los resultados académicos, es una realidad que a partir del hecho de vivir solo nacen diversos problemas, como el riesgo de afectación en la salud mental ${ }^{(17)}$, problemas de rutina alimenticia y la preocupación económica, ya que no cuentan con soporte familiar constante. No obstante, limitarse a la relación entre vivir solo y tener resultados académicos menos satisfactorios en los migrantes sería no observar todo el paradigma. La aculturación del estudiante migrante nacional e internacional es compleja y multifactorial, por ejemplo, se ha evidenciado que problemas frecuentes en este proceso son la barrera lingüística (especialmente en el lenguaje informal de la gente joven) y el apoyo insuficiente ${ }^{(18)}$. Además, es importante considerar el perfil académico escolar, ya que podría actuar como un factor protector para el fracaso académico.

En el contexto de este estudio, es importante mencionar al programa Beca 18. Desde su fundación (2011) tiene como objetivo la subvención de la educación superior, vivienda, alimentación, transporte, entre otras condiciones, de aspirantes jóvenes con buen rendimiento escolar en condición de pobreza o pobreza extrema ${ }^{(4)}$, adicionalmente, cuatro de diez becados son migrantes ${ }^{\left({ }^{19}\right)}$. A partir del 2019 se ha incorporado a la carrera de medicina humana como elegible. Es necesario considerar la implementación de programas de acompañamiento a estos estudiantes, evaluando su bienestar y los factores que podrían generar resultados académicos desfavorables, particularmente para aquellos estudiantes que viven solos.

El presente estudio presenta ciertas limitaciones. La muestra no es representativa en los países estudiados (solo fue censal en Perú) y solo se trabajó con estudiantes de primer y quinto año. Por otro lado, la definición de migrante puede clasificar incorrectamente los patrones migratorios, especialmente para los participantes de mayor edad o quienes se han mudado para estudiar el colegio y regresan a su ciudad de origen para estudiar la carrera. Sin embargo, estos casos son relativamente infrecuentes. No se realizó un análisis más preciso de la dinámica de la movilidad para estudiar, es decir de qué ciudades a qué ciudades se movilizaron, esta información puede ser valiosa para corroborar la hipótesis de que algunos estudiantes escogen provincias cercanas para migrar para no alejarse mucho de su familia.

En conclusión, el Perú tuvo la menor proporción de estudiantes de medicina humana migrantes, en comparación con los otros países latinoamericanos del estudio Red-LIRHUS. Aproximadamente, uno de cada cuatro estudiantes fue migrante, y hubo mayor proporción de estudiantes migrantes en universidades de provincia en comparación a universidades de Lima. Los migrantes tuvieron mayor probabilidad de vivir solos y de haber desaprobado cursos. Se debe evaluar la posibilidad de implementar programas o intervenciones en favor del bienestar de las personas que migran para su formación médica.

Contribuciones de autoría: PMT tuvo la idea de investigación y realizó el diseño de estudio y el análisis de datos. CEMM, DLC, FLJ, MOS y RPE participaron en la recolección de datos. PMT, DCM y RPE participaron en la redacción del manuscrito. CEMM, DLC, MOS, FLJ revisaron críticamente el manuscrito. Todos los autores aprobaron la versión final por publicar.

Fuentes de financiamiento: El presente estudio fue autofinanciado.

Conflictos de interés: RPE y CEMM migraron para estudiar la carrera de medicina.

\section{REFERENCIAS BIBLIOGRÁFICAS}

1. Willekens F, Massey D, Raymer J, Beauchemin C. International migration under the microscope. Science. 2016;352(6288):897-9. doi: 10.1126/science.aaf6545

2. Mendoza W. La inmigración venezolana en el Perú vista desde una perspectiva de salud. Rev Peru Med Exp Salud Publica. 2019;36(3):3812. doi: $10.17843 /$ rpmesp.2019.363.4812
3. Sanchez A. Migraciones internas en el Perú. Lima: Organización Internacional para las Migraciones; 2015

4. Cotler J. Educación superior e inclusión social. Un estudio cualitativo de los becarios del programa Beca 18 [Internet]. Lima: Pronabec; 2016 [citado el 24 de octubre del 2019]. Disponible en: https://www.pronabec. gob.pe/modPublicaciones/descarga/serie7_educacionsuperior.pdf 
5. Mayta-Tristán P, Pereyra-Elías R, Montenegro-Idrogo JJ, Mejia CR, Inga-Berrospi F, Mezones-Holguín E. Profile and professional expectations of medical students from 11 Latin American countries: the Red-LIRHUS project. BMC Res Notes. 2017;10(1):159. doi: 10.1186/s13104-017-2479-y

6. Pereyra-Elías R, Ocampo-Mascaró J, Silva-Salazar V, Vélez-Segovia E, da Costa-Bullón AD, Toro-Polo LM, et al. Prevalencia y factores asociados con síntomas depresivos en estudiantes de ciencias de la salud de una universidad privada de Lima, Perú 2010. Rev Peru Med Exp Salud Publica. 2010;27(4):520-6.

7. Zevallos-Morales A, Luna-Porta L, Medina-Salazar H, Yauri M, Taype-Rondan A. Association between migration and physical activity among medical students from a university located in Lima, Peru. PloS one. 2019;14(2):e0212009. doi: 10.1371/journal.pone.0212009

8. Kurré J, Scholl J, Bullinger M, Petersen-Ewert C. Integration and health-related quality of life of undergraduate medical students with migration backgrounds-Results of a survey. Psychosoc Med. 2011;8:Doc07. doi: 10.3205/psm000076

9. Huhn D, Resch F, Duelli R, Möltner A, Huber J, Jazi KK, et al. Examination performances of German and international medical students in the preclinical studying-term-A descriptive study. GMS Z Med Ausbild. 2014;31(3):Doc29. doi: 10.3205/zma000921

10. Rodríguez Gómez R. Migración de estudiantes: un aspecto del comercio internacional de servicios de educación superior. Papeles de Población. 2005;11(44):221-38.

11. Sosa ML. Migrantes en el sistema educativo argentino. Un estudio sobre la presencia de alumnos extranjeros en los estudios de nivel superior. Rev Iberoam Educ Sup. 2016;7(19):97-116.
12. Miñán W. Encuesta Anual de Costo de Vida 2019. Lima resultó la cuarta ciudad más cara para vivir en Sudamérica [Internet]. Perú: Gestión; 2019 [citado el 2 de julio del 2017]. Disponible en: https://gestion.pe/ economia/lima-resulto-cuarta-ciudad-cara-vivir-sudamerica-271367.

13. QS World University Rankings [Internet]. Reino Unido; 2019 [citado el 2 de julio del 2019]. Disponible en: https://www.qs.com/rankings/.

14. Lane R, Miranda R. The effects of familial acculturative stress and hopelessness on suicidal ideation by immigration status among college students. J of Am Coll Health. 2018;66(2):76-86. doi: 10.1080/07448481.2017.1376673

15. Diehl K, Jansen C, Ishchanova K, Hilger-Kolb J. Loneliness at universities: determinants of emotional and social loneliness among students. Int J Environ Res Public Health. 2018;15(9):1865. doi: 10.3390/ ijerph 15091865

16. Wilmoth JM, Chen P-C. Immigrant status, living arrangements, and depressive symptoms among middle-aged and older adults. J Gerontol B Psychol Sci Soc Sci. 2003;58(5):S305-S13. doi: 10.1093/geronb/58.5.s305

17. McManus I, Livingston G, Katona C. The attractions of medicine: the generic motivations of medical school applicants in relation to demography, personality and achievement. BMC Med Educ. 2006;6(1):11. doi: 10.1186/1472-6920-6-11

18. Huhn D, Junne F, Zipfel S, Duelli R, Resch F, Herzog W, et al. International medical students-a survey of perceived challenges and established support services at medical faculties. GMS Z Med Ausbild. 2015;32(1):Doc9. doi: 10.3205/zma000951

19. Programa Nacional de Becas y Crédito Educativo (Pronabec). Estadísticas: Becas otorgadas. Beca 18 pregrado 2015 [Internet]. Perú: Pronabec; 2015 [citado el 20 de octubre del 2019]. Disponible en: http://www.pronabec. gob.pe/modPublicaciones/descarga/anuario2016-pregrado-beca18.pdf 